\title{
Informed Consent for Surgery During COVID-19
}

\author{
Neela Bhattacharya ${ }^{1} \cdot$ Kaushik Bhattacharya ${ }^{2}$ (C) \\ Received: 16 April 2020 / Accepted: 24 April 2020 / Published online: 8 May 2020 \\ (C) Association of Surgeons of India 2020
}

\begin{abstract}
Informed consent has become a challenging issue when surgery at the time of novel corona virus disease 2019 (COVID-19) is to be performed, in view of increased risk of the need of intensive care unit (ICU) in the post-operative period and the associated high mortality if a patient exhibits COVID-19 symptoms in the post-operative period. We have devised a new informed consent format for all patients undergoing surgery incorporating a few points specific for the disease.
\end{abstract}

Keywords COVID-19 $\cdot$ Consent $\cdot$ Pandemic

\section{Introduction}

Informed consent for surgery, which is a critical component of surgical practice, has become a challenging issue in the time of COVID-19 infection. There are few specific requirements which need to be discussed pre-op during this pandemic with the patient and the relatives, in order to avoid any unnecessary medicolegal issues. We have devised the first pro forma of informed consent of such patients undergoing surgical intervention.

\section{Informed Consent During COVID-19}

On March 11, 2020, the World Health Organization (WHO) declared the novel corona virus disease 2019 (COVID-19) a global pandemic, which classifies the outbreak as an international emergency. In a retrospective cohort study of 34 operative patients with confirmed COVID-19, $44.1 \%$ of patients required ICU care in the post-operative period and mortality rate was $20.5 \%$ [1]. Therefore, it is very important to devise a separate informed consent for operative patients during COVID-19,

Kaushik Bhattacharya

kbhattacharya10@yahoo.com

1 Anandaloke Multispeciality Hospital, Siliguri, West Bengal 734001, India

2 CAPFs Composite Hospital Border Security Force Kadamtala, Siliguri, West Bengal 734010, India taking all the risk under consideration. We are reporting the first ever informed consent pro forma especially designed for COVID-19 patients undergoing surgery.

\section{Consent Form}

\section{Discussion}

The Canadian Medical Protective Association reports that over a recent 5-year period, $65 \%$ of medicolegal cases involving informed consent were surgical and only $21 \%$ of these cases were decided in favour of the surgeon [2]. It is mandatory that prior to obtaining consent for the proposed surgery, the surgeon must provide the patient with detailed information about the nature of the surgery, the expected benefits, alternate treatments and the consequence of not having the surgery [3].

In a country like India, the need for ICU in the postop period must be discussed with the patient's relative as it involves an increased financial burden. There are also concerns about the lack of pre-op COVID testing in India due to paucity of commercial testing kits. A normal patient may develop or contract corona virus infection in the peri- or post-operative period which should be again documented in the consent form. Hence, a detailed modified consent form was designed exclusively for all patients who require surgery during this pandemic. 
It has been explained to me in a language I understand that patient

.... years of age, Hospital ID No / Aadhar No: with the diagnosis of has been admitted under Dr.... for emergency/elective surgery during the COVID 19 pandemic. In this time, every hospital is considered a high risk zone for transmission of Corona SARS virus. Therefore, any surgical procedure carries additional risk, as the patient, patient party, hospital staff or the treating doctor may be an asymptomatic carrier or an unsuspected COVID patient and there is a chance of the surgical patient contracting the illness in the post- operative period. That may entail prolonged hospitalisation, ICU transfer and care and a $20 \%$ increased risk to life in these circumstances. At the present time, there is /there is no provision for COVID testing in this hospital.

I have agreed to treatment in this hospital, after having understood these facts. I also give an undertaking that $\mathrm{I} / \mathrm{my}$ patient does not have any signs and symptoms of the SARS COVID infection (fever, cough, difficulty in breathing, loss of taste sensation and /or loss of smell) at present. I hereby understand that the doctor will take all possible precautions to prevent such an infection from happening. I promise to follow all protocols prescribed for the safety of the patient by him/her. I have also been counselled regarding the additional cost of protective equipment required. I hereby authorise Dr...... and her/his team to operate on the patient. In event of the patient or party contracting the Corona SARS Virus infection, even after all protocols are followed, I will not hold the doctor, the hospital or its staff morally or legally accountable for the same.

This is to state that I have comprehensively explained the information above along with the discussion/explanations provide by the doctors, to the patient and/or his/her attendant in a way which I believe he/she/they can understand and the patient and/or his/her attendant have informed me that they have understood the foresaid information completely.

Name:

Contact No:

Date:

Time:
Name:

Contact No:

Signature of Surgeon

Name and Designation: 


\section{Compliance with Ethical Standards}

Conflict of Interest The authors declare that they have no conflict of interest.

\section{References}

1. Lei S, Jiang F, Su W, Chen C, Chen J, Mei W, Zhan LY, Jia Y, Zhang L, Liu D, Xia ZY, Xia Z (2020) Clinical characteristics and outcomes of patients undergoing surgeries during the incubation period of COVID-19 infection. EClinicalMedicine 21:100331. https://doi. org/10.1016/j.eclinm.2020.100331

2. Hanson M, Pitt D (2017) Informed consent for surgery: risk discussion and documentation. Can J Surg 60:69-70

3. Canadian Medical Protective Association. Risk Fact Sheet CMPA. [accessed 2016 Mar. 1]. Available: www.cmpa-acpm.ca/documents/ 10179/300031190/informed_consent-e.pdf

Publisher's Note Springer Nature remains neutral with regard to jurisdictional claims in published maps and institutional affiliations. 